\title{
Silla bipedestadora para personas con movilidad restringida grado IV en las extremidades inferiores con una capacidad de carga de $100 \mathrm{~kg}$
}

\section{Standingchair for people with restricted mobility grade IV at the lower limbs with a load capacity of $100 \mathrm{~kg}$}

\section{Cadeira bipedestadora para pessoas com movimentação de grade IV restringida na terra baixa com uma capacidade de carga de $100 \mathrm{~kg}$}

\author{
Fabio Eduardo Obando-Herrera', Tatiana Gabriela Flores-Mugmal², Jhonny Israel Barbero-Palacios ${ }^{3}$ y \\ Luis Alexis Ortega-Bustamante ${ }^{4}$
}

Forma de citar: F.E. Obando-Herrera, T.G. Flores-Mugmal, J.I. Barbero-Palacios y L.A. Ortega-Bustamante, "Silla bipedestadora para personas con movilidad restringida grado IV en las extremidades inferiores con una capacidad de carga de 100kg", Respuestas, vol. 22, no. 2, pp. 76-86, 2017.

Recibido:

Enero 28 de 2017

Aceptado:

Mayo 13 de 2017

\section{Resumen}

El presente artículo describe el proceso de diseño y construcción de una silla de ruedas capaz de permitir la posición de bipedestación a pacientes con discapacidad grado IV en miembros inferiores, de hasta $100 \mathrm{~kg}$ de peso. Para el dimensionamiento de la silla se consideró un análisis antropométrico a 19 pacientes adultos (13 mujeres y 6 hombres) del Centro de Rehabilitación Integral Especializado CRIE Nro. 1, ubicado en la parroquia Conocoto - Quito, Ecuador, se utilizan los percentiles P95 de las mediciones antropométricas de los pacientes del centro. Usando el software Autodesk Force Effect, se simuló el movimiento del paciente al pasar de sedestación a bipedestación, y así controlar posiciones relativas de miembros inferiores y tronco, en un rango de movimiento desde $15^{\circ}$ a $75^{\circ}$, y tener una base de datos para la selección del sistema impulsor en la silla. Mediante un análisis estático de cargas en el cuerpo del paciente, se designó las cargas críticas de diseño para los miembros críticos de la silla, los mismos que fueron comprobados mediante un análisis de esfuerzos con el software Autodesk Inventor V15. También se expone las pruebas y evaluación supervisada por 10 profesionales terapistas físicos, mediante pruebas in situ con pacientes del CRIE $\mathrm{N}^{\circ} 1$ y con una encuesta a los profesionales terapistas físicos.

Palabras Clave: Antropometría, Bipedestación, Discapacidad, Silla tfloresm@est.ups.edu.ec Universidad Politécnica

Salesiana Quito, Ecuador

${ }^{3}$ Ingeniero Mecánico jbarbero@est.ups.edu.ec Universidad Politécnica Salesiana Quito, Ecuador

${ }^{4}$ Ingeniero Mecánico lortegab@est.ups.edu.ec Universidad Politécnica

Salesiana Quito, Ecuador

\begin{abstract}
This paper describes the design and construction process of a wheelchair capable of allowing standing position to patients with grade IV disability in lower limbs, up to 100 $\mathrm{kg}$ weight, located in the Conocoto parish - Quito, Ecuador, the P 95 percentiles of the anthropometric measurements of the patients of the center. Using Autodesk Force Effect software, the patient's movement was simulated by moving from sitting to standing, and thus controlling relative positions of lower limbs and trunk, in a range of motion from $15^{\circ}$ to $75^{\circ}$, and having a database for the selection of the drive system in the chair. Through a static analysis of loads on the patient's body, critical design loads were assigned to critical chair members, which were tested through stress analysis with Autodesk Inventor V15 software. We also present the tests and evaluation supervised by 10 physical therapists professionals,
\end{abstract}


Fabio Eduardo Obando-Herrera, Tatiana Gabriela Flores-Mugmal, Jhonny Israel Barbero-Palacios y Luis Alexis Ortega-Bustamante

through in situ tests with patients of CRIE $\mathrm{N}^{\circ} 1$ and with a survey to the physical therapist professionals

Keywords: Anthropometry, Standing, Disability, Chair

\section{Resumo}

O presente artigo descreve o processo de projeto e construção de uma cadeira de rodas capaz de permitir a posição de pé em pacientes com deficiência de grau IV em membros inferiores de até $100 \mathrm{~kg}$ de peso. Para dimensionar a cadeira de uma análise antropométrica considerou 19 pacientes adultos (13 mulheres e 6 homens) Centro de Reabilitação Integral Especializada do CRIE No. 1, localizado na freguesia Conocoto -. Quito, Equador, os percentis P95 de usados Medições antropométricas de pacientes no centro. Usando o software Autodesk Force Effect, o movimento do paciente foi simulado, movendo-se de um lado para outro, e assim controlando as posições relativas dos membros inferiores e do tronco, em uma amplitude de movimento de $15^{\circ}$ a $75^{\circ}$, e tendo um banco de dados para a seleção do sistema de acionamento na cadeira. Através de uma análise estática de cargas no corpo do paciente, as cargas críticas do projeto foram atribuídas aos membros críticos da cadeira, que foram testados através de análise de estresse com o software Autodesk Inventor V15. Os testes e avaliação supervisionados por 10 fisioterapeutas também são apresentados, através de testes no local com CRIE N - 1 e uma pesquisa de profissionais de fisioterapia.

Palavras-chave: Antropometria, Permanente, Deficiência, Cadeira

\section{Introducción}

Una persona con capacidades especiales o discapacidad [1], [2], es aquella que, como consecuencia de una o más deficiencia o pérdida o anormalidad de una estructura $o$ función, como física, mental, intelectual o sensorial [3], sin importar la causa que lo hubiere originado, ve restringida permanentemente su capacidad biológica, psicológica y asociativa para ejercer una o más actividades esenciales de la vida diaria.

\section{El Centro de Rehabilitación Integral y} Especializado (CRIE Nro. 1) ubicado en la parroquia de Conocoto - Quito, Ecuador, brinda el servicio de rehabilitación en el área de fisioterapia, este centro cuenta con los espacios adecuados y máquinas especializadas para brindar tratamiento a los diferentes tipos de discapacidad, pero no cuenta con un bipedestador eléctrico tipo silla de ruedas, para sus terapias.

En Ecuador se adoptó el instrumento internacional para la valoración de las situaciones de minusvalía conformada por Baremos, Estos baremos establecen normas para la evaluación de las consecuencias de la enfermedad, de acuerdo con el modelo propuesto por la clasificación internacional de deficiencias, discapacidades y minusvalías de la O.M.S [4], entonces se considera a un paciente con discapacidad grado IV, a la persona con discapacidad grave ya que las secuelas imposibilitan la realización de la mayoría de las actividades diarias generales [5]. 
Los pacientes observados, presentan distintas patologías como: fractura T12 $(5 \%)$, reumatismo $(5 \%)$, lesión ciática $(5 \%)$, osteoartritis $(5 \%)$, flacidez muscular $(5 \%)$, lumbalgia $(5 \%)$, operación tumoral $(5 \%)$, traumatismo $(5 \%)$, coxartrosis $(5 \%)$, trombosis $(5 \%)$, artrosis medular $(5 \%)$, hemiplejia (11\%), hemiparesia (32\%), todas las patologías antes mencionadas son consideradas como discapacidad grado IV y requieren el uso de silla de ruedas [6].

\section{Materiales y Métodos}

\subsection{Análisis Antropométrico}

La antropometría se ocupa del estudio de las dimensiones estructurales y funcionales del cuerpo humano, siendo las medidas más usuales la longitud, el peso y tipo de movimiento [7].

Son varios factores que influyen en las dimensiones anatómicas tales como: la edad, el sexo, la raza, la ocupación, la vestimenta e incluso la hora del día [8].

Las dimensiones estructurales o estáticas, que se toman en posiciones fijas estandarizadas (puntos de referencia esqueléticos anatómicos), que se ubican al palpar las prominencias óseas [7], [9].

\subsection{Proceso antropométrico}

Los datos antropométricos se obtuvieron de 19 pacientes (13 mujeres y 6 hombres) con edades de $52 \pm 18$ años en los hombres y $70 \pm 9$ años en mujeres, quienes requieren el uso de silla de ruedas, registrados en la base de datos CRIE Nro. 1, los mismos que recibieron información suficiente y dieron su aprobación por escrito, para colaborar en la investigación, la misma que fue financiada en su totalidad por la Universidad Politécnica Salesiana.

\subsubsection{Medidas antropométricas}

En el modelo biomecánico figuras 1 y 2 se detalla el código de la medida antropométrica [10], con los que se utilizó en la investigación:

Las mediciones antropométricas (figura 1) (figura 2) se las realizaron con la colaboración de dos personas un medidor y un anotador, quienes tienen el adiestramiento requerido para la toma de mediciones, los instrumentos utilizados fueron: lápiz demográfico, cinta métrica, kit antropométrico profesional CESCORF, los mismos que tenían el respectivo certificado de calibración.

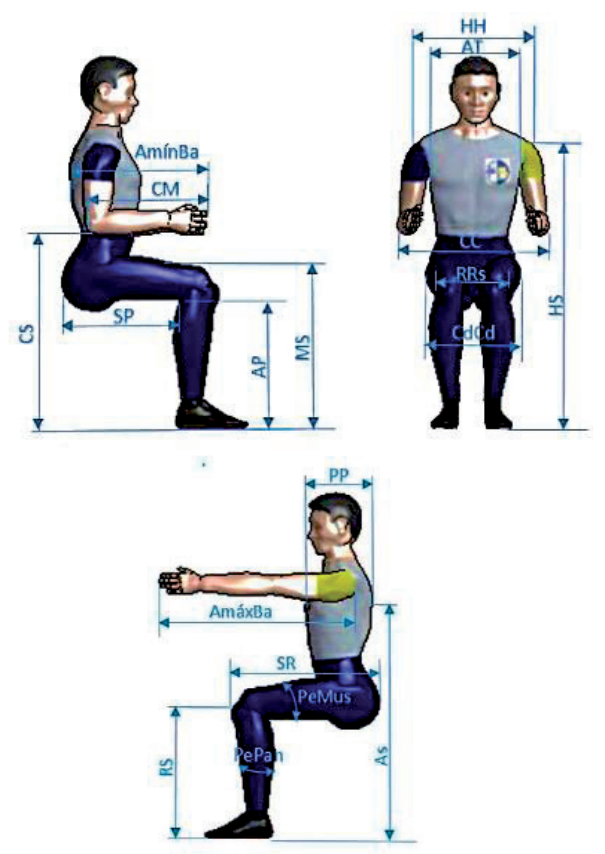

Figura 1: Mediciones antropométricas - sentado.

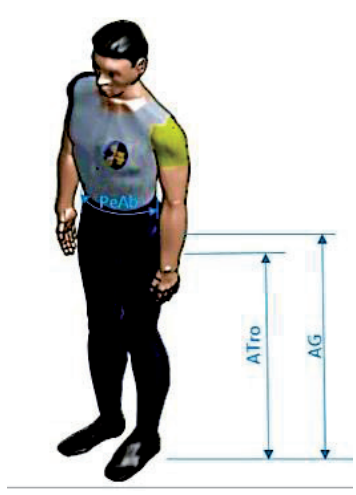

Figura 2. Mediciones antropométricas - parado.

El análisis estadístico se realizó mediante el software Microsoft Excel 2013, utilizando los criterios antropométricos a los percentiles 
Fabio Eduardo Obando-Herrera, Tatiana Gabriela Flores-Mugmal, Jhonny Israel Barbero-Palacios y Luis Alexis Ortega-Bustamante

extremos de la población [11], por lo que en las tablas I se muestra los resultados del cálculo del percentil 95, que es el más empleado en el diseño ergonómico y que facilitan las relaciones funcionales de la silla [10], [12], [13].

Tabla I. Percentiles hombres (H) - mujeres (M).

\begin{tabular}{|c|c|c|c|c|}
\hline CÓDIGO & MEDIA (CM) & DESV. EST. & P95 & P95 (M) \\
\hline AP & 42,85 & 1,940 & 44,72 & 42,82 \\
\hline SP & 43,1 & 4,665 & 47,64 & 46,84 \\
\hline SR & 54,7 & 4,718 & 59,8 & 53,42 \\
\hline MS & 55,05 & 1,913 & 56,44 & 54,28 \\
\hline RS & 53,05 & 2,424 & 55,66 & 51,9 \\
\hline CS & 62,45 & 2,073 & 63,8 & 67,2 \\
\hline AmínBa & 52,4 & 2,688 & 57,8 & 54,12 \\
\hline CM & 42,2 & 4,285 & 47,3 & 45,78 \\
\hline AmáxBa & 73,5 & 6,783 & 82 & 74,2 \\
\hline HS & 95 & 1,800 & 98,2 & 97,8 \\
\hline CdCd & 34 & 4,725 & 40,88 & 35,4 \\
\hline RRs & 23,1 & 1,906 & 26,08 & 26,78 \\
\hline AS & 87 & 2,973 & 89,98 & 89 \\
\hline CC & 50,75 & 6,536 & 53,88 & 52,7 \\
\hline PP & 27 & 1,837 & 28,54 & 33,38 \\
\hline HH & 39,35 & 2,214 & 40,74 & 41,36 \\
\hline AT & 31,8 & 2,561 & 33,64 & 34 \\
\hline PeMus & 45 & 6,917 & 54 & 48,4 \\
\hline PePan & 35,25 & 2,840 & 38,7 & 37,4 \\
\hline Atro & 85,5 & 5,492 & 91,4 & 88,2 \\
\hline AG & 97 & 6,031 & 100 & 95 \\
\hline PeAb & 101,5 & 14,680 & 127,8 & 110 \\
\hline
\end{tabular}

\subsection{Dimensionamiento general de la silla}

Tomando en cuenta las diferencias antropométricas entre géneros, siendo los hombres los que presentan dimensiones mayores [14], [15], se procedió a dimensionar los espacios y distancias de la silla usando los valores del $\mathrm{P}_{95}[16]$, [17], más el espacio de tolerancia [18], lo que garantiza que al menos $95 \%$ de la población será capaz de usar la silla sin molestia [19], [20],También se tomó en cuenta las posturas de los pacientes en estudio [20], en la figura 3 se detallan las medidas en centímetros (Figura 3).
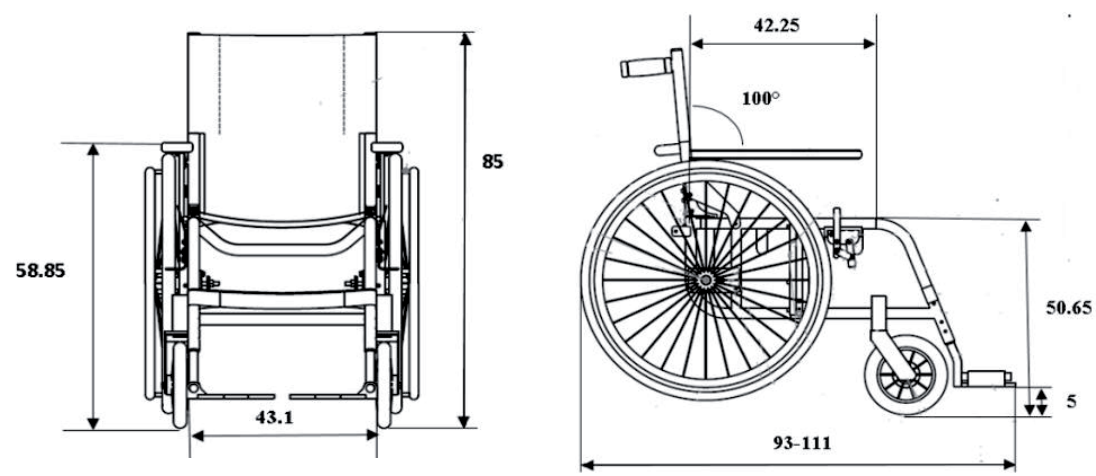

Figura 3. Dimensiones finales de la silla. 


\subsection{Alternativas del sistema de elevación}

Se planteó tres alternativas, silla con sistemas hidráulico, mecánico (tornillo de potencia) y eléctrico, mediante un análisis cualitativo [21] y cuantitativo de ponderación simple, tomando en cuenta los siguientes parámetros: facilidad de accionamiento, uso de fuerza muscular, mantenimiento, facilidad de montaje, costos, existencia de materiales en el mercado, los mismos que se ponderaron excelente (5); muy bueno (4); bueno (3); regular (2); malo (1). Se seleccionó sistema de elevación eléctrico, el mismo que se detalla en la figura 4.



Figura 4. Silla con sistema de elevación eléctrico.

\subsection{Selección Actuador lineal}

Como unidad de fuerza de empuje se consideró un actuador lineal Dewert modelo megamat 2, con una carga de empuje de 600 $\mathrm{N}$, velocidad máxima de $28 \mathrm{~mm} / \mathrm{s}$, longitud de carrera máxima de $425 \mathrm{~mm}$ y voltaje de entrada $110 \mathrm{~V}$ [22].

\subsection{Análisis de un paciente en sedestación y bipedestación}

La posición bípeda forma parte de la herencia humana, proporcionando bienestar, beneficia al sistema circulatorio [23], reduciendo la espasticidad [24]. Al adquirir la posición bípeda el paciente logra extender las articulaciones de los miembros inferiores, previniendo contracturas en los mismos [23] [25].
La verticalidad mejora la atención y facilita las interacciones sociales, poniendo al paciente a nivel de los ojos de otras personas [26].

En la postura de pie, el menor uso de energía se produce cuando la línea vertical de la gravedad cae a través de la columna ósea de sostén. Si la línea de gravedad pasa por el centro de cada articulación se aplica menos tensión sobre músculos y ligamentos [18].

Se considera a la rodilla como la articulación más grande del esqueleto humano, la misma que soporta la mayor parte del peso del cuerpo en posición de bipedestación, en ella se unen el fémur, la tibia y la rótula [27], esta articulación presenta una superficie cóncava que se desliza sobre otra convexa alrededor de 2 ejes, existen dos cuerpos que sirven como amortiguadores entre el fémur y la tibia, denominados los meniscos femorales [28], [29], desde el punto de vista morfológico la rodilla constituye la articulación encargada de la flexión y extensión de la pierna [30], [31]. La articulación de la rodilla realiza fundamentalmente movimientos en 2 planos perpendiculares entre sí (planos sagital $\mathrm{y}$ frontal) [27], posee un fuerte aparato ligamentoso, cuya función es darle estabilidad y evitar movimientos excesivos [28], [29].

De investigaciones realizadas se registra el par máximo de extensión de la rodilla cuyo valor es de $0.38 \mathrm{~N} . \mathrm{m} / \mathrm{kg}$ aproximadamente (30.4 N.m para una persona de $80 \mathrm{~kg}$ ) [32].

Los miembros inferiores fueron representados como un mecanismo plano formado por eslabones simples, en el cual la articulación de la rodilla, fue considerado como eslabón crítico [27], también se incluyó a otros segmentos del cuerpo humano: cuello, tronco, cadera, pierna y pie, representados en forma de eslabones.

Para la simulación se utilizó el programa Autodesk Force Effect, en el que se introdujeron las medidas del $\mathrm{P}_{95}$, considerando 
a la pantorrilla (EF) como un elemento fijo, por efecto del movimiento que produce la silla bipedestadora, el muslo (BC) gira un ángulo de hasta $75^{\circ} \mathrm{y}$ el conjunto cadera (CD) $\mathrm{y}$ tronco, tienen un desplazamiento sobre el eje vertical como se muestra en las figuras 5 .
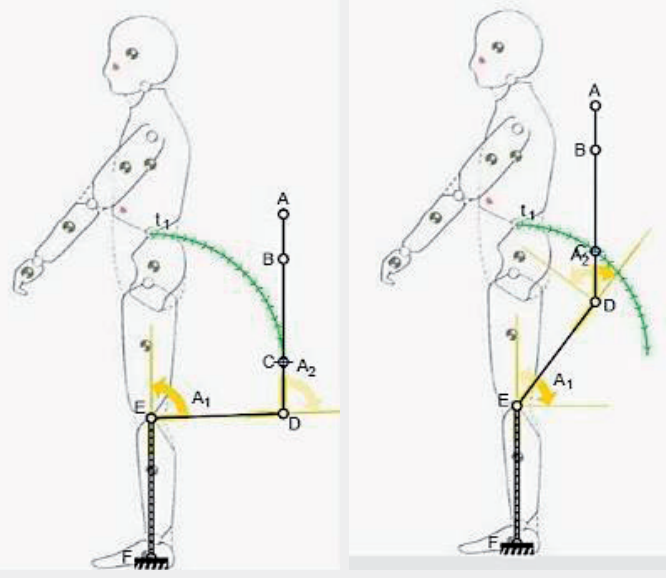

Figura 5. Simulación posición inicial - final Autodesk Force Effect.

En el software se ingresó diferentes valores de velocidad angular y se observó el movimiento del sistema, a los datos obtenidos, se los relacionó con la energía cinética rotacional total [33].

El momento de inercia del muslo se lo obtuvo modelándolo en Autodesk Inventor Professional 2015, con las dimensiones Ds = $171,1 \mathrm{~mm}, \mathrm{Di}=132,9 \mathrm{~mm}$ y SP $476,4 \mathrm{~mm}$. Obteniendo el momento de inercia 174500 kg. $\mathrm{mm}^{2}$.

Sin embargo, el software no está considerando las patologías de los pacientes, es decir en las simulaciones se observa que los eslabones siguen una trayectoria ordenada y soportan la velocidad angular fijada por el software, por lo que es recomendable considerar que los bipedestadores presenten movimientos suaves y lentos.

Por lo mencionado anteriormente, la velocidad lineal del actuador debe ser aproximadamente $\mathrm{v}=8 \mathrm{~mm} / \mathrm{s}$, equivalente a $0,0168 \mathrm{rad} / \mathrm{s}$, esta velocidad se ejerce sobre la estructura del bipedestador y por ende a los muslos por lo que aplicando en la ecuación (1), obteniendo que la energía cinética rotacional de los dos muslo es $0,05 \mathrm{~J}$.

Por lo que se concluye que el actuador seleccionado proporciona la energía cinética necesaria para que el paciente pase de la posición de sedestación a bipedestación, con la mayor comodidad y ningún esfuerzo de sus músculos.

Para la estructura se seleccionó acero ASTM A-36, considerando sus propiedades mecánicas como resistencia y soldabilidad que superan a materiales como aluminio y acero inoxidable [34], posee buenas características en lo referente a durabilidad, su susceptibilidad a la corrosión la evitamos con el uso de recubrimientos de pintura anticorrosiva, su costo es relativamente bajo y su gran stock en el mercado local, hace que se trabaje con este material.

\subsection{Determinación de cargas de trabajo}

Tomando en cuenta el peso del tronco y los muslos, considerando una carga máxima de diseño $100 \mathrm{~kg}$. (980.67 [N]), se toma en cuenta la tabla de pesos segmentarios [35], se calculó las cargas de trabajo las mismas que se detallan en la tabla II:

Tabla II. Pesos segmentarios, tronco y muslo.

\begin{tabular}{|c|c|c|}
\hline MIEMBRO & \% DEL PESO & PESO [N] \\
\hline Tronco & $50.7 \%$ & 497.2 \\
\hline Muslo & $10,3 \%$ & 101.0 \\
\hline
\end{tabular}

La ubicación de las cargas de trabajo se detallan en el modelo biomecánico de la figura 6 .
ISSN 0122-820X

E-ISSN 2422-5053

PP: $76-86$ 
Silla bipedestadora para personas con movilidad restringida grado IV en las extremidades inferiores con una capacidad de carga de $100 \mathrm{~kg}$

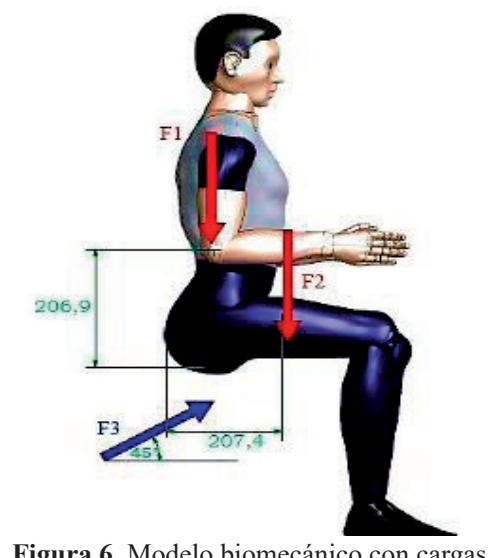

Figura 6. Modelo biomecánico con cargas

Donde:

$\mathrm{F}_{1}$ : fuerza segmentaria en el centro de gravedad de la espalda

$\mathrm{F}_{2}$ : fuerza segmentaria en el centro de gravedad del muslo

$\mathrm{F}_{3}$ : fuerza del actuador lineal

Aplicando las ecuaciones de equilibrio [36] se obtiene $\mathrm{F}_{3}=845.99[\mathrm{~N}]$, que es la fuerza necesaria para levantar el peso segmentario del tronco y el muslo, con lo que se comprueba que el actuador lineal seleccionado trabaja sin inconvenientes con la carga máxima de diseño.

\subsubsection{Diseño de soportes de asiento (nodo crítico)}

La fuerza neta de diseño $980[\mathrm{~N}](100 \mathrm{~kg}$.) se dividió para los dos soportes, obteniendo una carga $490[\mathrm{~N}]$ y una longitud de $422.50 \mathrm{~mm}$ para el elemento crítico mostrado, se decidió aplicar un factor de seguridad de diseño, $n=2$ [37] (Figura 7).


Figura 7. Cargas en el soporte del asiento.
Se comprobó si la estructura no falla por deflexión, usando el método de deflexión admisible [38] aplicando la ecuación (1). Obteniendo una deflexión admisible de $1,21 \mathrm{~mm}$ que es mayor a la obtenida en la simulación, con lo que comprobamos que el nodo crítico no falla por deflexión.

$$
[\mathrm{v}]=\frac{\mathrm{L}}{350}
$$

En cuanto a la estructura, debido a su compleja geometría el análisis se realizó con el entorno de análisis estático en Autodesk Inventor Profesional 2015. Se aplicó la carga máxima de trabajo de $980 \mathrm{~N}$ obteniendo una deflexión máxima de 0.133mm (Figura 18).

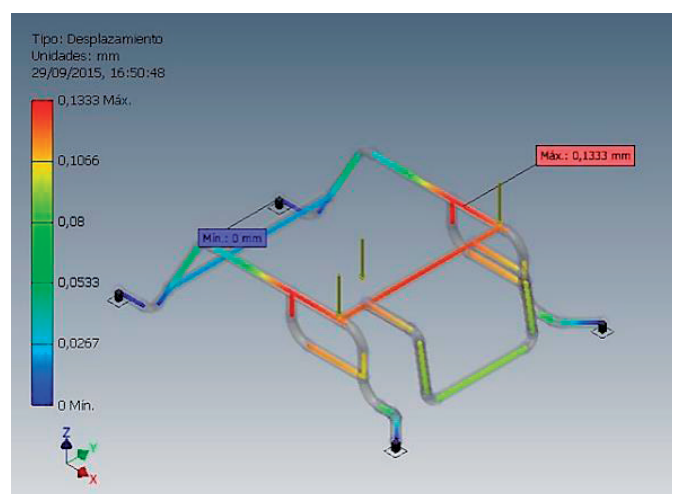

Figura 8. Simulación de la estructura-Desplazamientos.

\section{Resultados y análisis}

\subsection{Estructura de la silla}

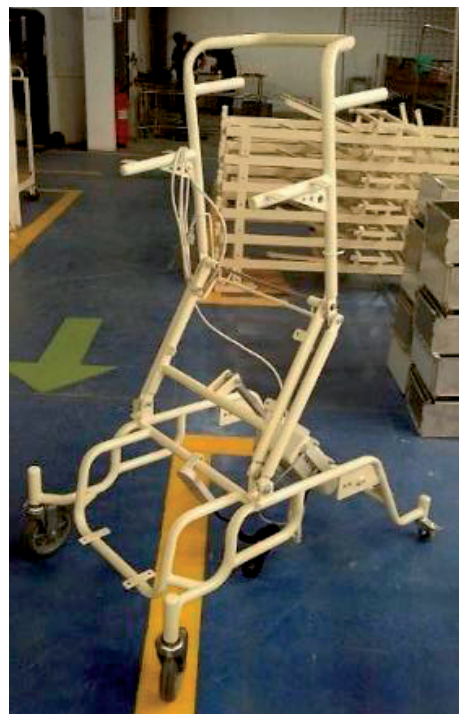

Figura 9. Silla en construcción. 


\subsection{Evaluación}

La evaluación se llevó acabo en el CRIE $\mathrm{N}^{\circ} 1$, con la presencia de diez profesionales terapistas físicos, quienes laboran en el mencionado centro (Figura 10) (Figura 11).

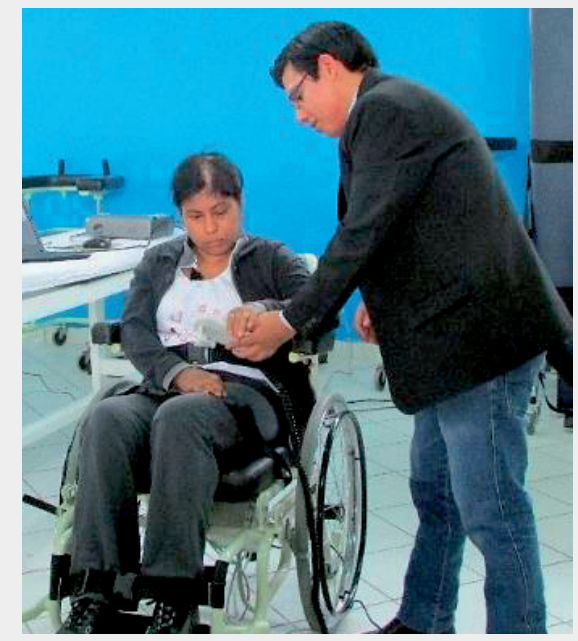

Figura 10. Prueba posición sedestación.

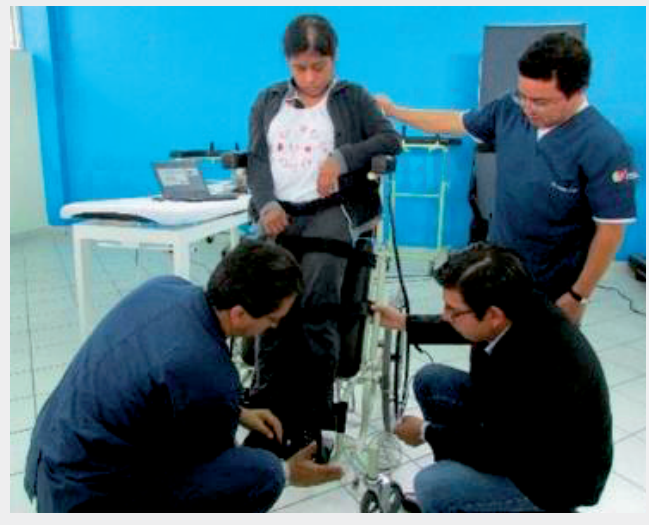

Figura 11. Prueba posición bipedestación.

La evaluación la hicieron todos los profesionales terapistas físicos, mediante una encuesta personal y por escrito, con un criterio de evaluación de 1 a 10 y emitieron sugerencias individualmente, en la encuesta se evaluó los siguientes parámetros: facilidad de manipulación, facilidad de operación, estética, funcionalidad, facilidad de transporte obteniendo los siguientes resultados:

Tabla III. Evaluación terapistas físicos.

\begin{tabular}{|c|c|c|c|c|c|c|c|c|c|c|}
\hline SUGERENCIAS DE CAMBIO & E1 & E2 & E3 & E4 & E5 & E6 & E7 & E8 & E9 & E10 \\
\hline Facilidad de manipulación & 5 & 9 & 8 & 7 & 10 & 9 & 7 & 9 & 9 & 9 \\
\hline Facilidad de operación & 4 & 10 & 8 & 8 & 9 & 9 & 8 & 10 & 10 & 10 \\
\hline Estética & 3 & 8 & 8 & 7 & 9 & 9 & 8 & 9 & 9 & 9 \\
\hline Funcionalidad & 6 & 9 & 8 & 8 & 9 & 9 & 7 & 10 & 9 & 10 \\
\hline Facilidad de transporte & 2 & 9 & 7 & 8 & 8 & 9 & 7 & 9 & 9 & 9 \\
\hline Promedio & 4 & 9 & 7,8 & 7,6 & 9 & 9 & 7,4 & 9,4 & 9,2 & 9,4 \\
\hline
\end{tabular}

Como se expresa en la tabla III, la calificación promedio de 6 evaluadores es 9,17 con lo que se puede concluir que no sugieren cambios para la silla, la calificación promedio de 3 evaluadores es de 7,6 con lo que se puede concluir que sugieren cambios mínimos y solamente 1 evaluador califica a la silla con 4 sugiriendo cambios.

Siendo el presente proyecto financiado en su totalidad por la Universidad Politécnica Salesiana, Quito - Ecuador, como proyecto de investigación de fondos concursables, las sugerencias emitidas por los evaluadores terapistas físicos, se tomarán en cuenta para el rediseño de la silla bipedestadora en un futuro proyecto.

\section{Conclusiones}

Se presentó el diseño mecánico y construcción de una silla de ruedas bipedestadora para en una persona con movilidad restringida grado IV, en sus miembros inferiores, con el objetivo principal de mejorar su calidad de vida. La silla bipedestadora cumple con 
todos los requerimientos de resistencia mecánica, control de movimiento, facilidad de operación, facilidad de transporte y un mantenimiento reducido.

Adicionalmente la silla podrá ser usada como equipo de rehabilitación física para pacientes del Centro de Rehabilitación Integral Especializado CRIE Nro. 1, ubicado en la parroquia Conocoto - Quito, Ecuador.

Para los parámetros iniciales de diseño se obtuvo mediante un estudio antropométrico de un grupo de 19 pacientes adultos (13 mujeres y 6 hombres) del CRIE Nro. 1, quienes presentan un grado IV de discapacidad en sus miembros inferiores. El análisis estadístico se realizó mediante el software Microsoft Excel 2013, utilizando los criterios antropométricos a los percentiles extremos de la población. Con los datos obtenidos se dimensionó la geometría preliminar de la silla bipedestadora que fue la base para el estudio de cargas y el diseño mecánico.

La asistencia para la bipedestación se hace a través de un actuador lineal eléctrico Dewert modelo megamat 2, el mismo que cumple con todos los requerimientos referentes a velocidad de elevación, carga a elevar, económico, fácil manipulación y existe en el mercado local.

En este proyecto se utilizó herramientas informáticas como Autodesk Inventor v15, Autocad Mechanical v15, Autodesk Force Effect, ya que permitieron el uso de modelos en $2 \mathrm{D}$ y $3 \mathrm{D}$ y con un análisis paramétrico se logró totalizar todas las restricciones geométricas del diseño estructural de la silla bipedestadora, con ellos se pudo examinar diferentes configuraciones de los componentes y la silla.

Las evaluaciones hechas por los profesionales fisioterapistas del CRIE $\mathrm{N}^{\circ} 1$, indican que el $60 \%$ de los evaluadores mencionan que la silla bipedestadora cumple con las características necesarias para las que fue construida, el $30 \%$ manifiesta que se deben hacer mínimas modificaciones a la silla y solamente un evaluador sugiere cambios obligatorios. No obstante a este proyecto se le considera como piloto para desarrollar futuras innovaciones y obtener mejores e innovadores productos.

\section{Recomendaciones}

La silla bipedestadora está diseñada para superficies planas, por lo que se recomienda proporcionar el acompañamiento necesario de una o dos personas si se la usa en superficies inclinadas.

No exceder el peso máximo de $100 \mathrm{~kg}$, que se ha establecido en el diseño de la silla bipedestadora, ya que esto provocará deformaciones en la estructura o elementos que son vitales para su funcionamiento.

\section{Referencias}

[1] M.R.L y CONADIS, Manual de buenas prácticas para la inclusión laboral de personas con discapacidad, Quito, Ecuador: MRL, 2013.

[2] W. Momm y R. Ransom, Enciclopedia de salud y seguridad en el trabajo. Madrid: Instituto Nacional de Salud e Higiene en el Trabajo, 2000.

[3] C. Egea, A. Sarabia, "Clasificaciones de la OMS sobre discapacidad", Boletín del Real Patronato sobre Discapacidad, no. 50, pp. 15-30, 2011.

[4] Ministerio de Salud Pública, 2015. [En línea]. Available: www.salud.gob.ec. [Accedido: 23-oct-2016]

[5] "Valoración de las situaciones de discapacidad". [En línea]. Available: http://www. juntadeandalucia.es/export/ drupaljda/valoracion_discapacidades. pdf. [Accedido: 30-jul-2016]

[6] Instituto de Migraciones y Servicios 
Sociales, Valoración de las situacionesde minusvalía, Madrid: Grafo S.A, 2000.

[7] R.R. Rivas, Ergonomía en el diseño y la producción industrial, Buenos Aires: Nobuko, 2007.

[8] S.A. Konz, Diseño de sistemas de trabajo, Mexico: Limusa, 1992.

[9] W. Laurig y J. Vedder, Ergonomía. en enciclopedia de la OIT, D - INSHT (Instituto Nacional de Seguridad e Higiene en el Trabajo), 2012.

[10] Mondelo, Torada, Busquets y Bombardó, Diseño de puestos y espacios de trabajo, Barcelona: Alfaomega, 2000.

[11] M. Vergara Monedero, «Evaluación Ergonómica de Sillas. Criterios de Evaluación basadosenla Postura,» Enero 1998. [En línea]. Available: http://www. tdx.cat/bitstream/ handle/10803/10560/ vergara. pdf? sequence $=1$ [Accedido: 15-may-2016]

[12] E. Valero, "Antropometría", Instituto Nacional de seguridad e Higiene del Trabajo, Madrid, 2012.

[13] J.L. Devore, Probabilidad y Estadistica para Ingeniería y Ciencias, México: Cengage Learning, 2008.

[14] P. Pelayo, M. Sidney, T. Kherif, D. Chollet y C. Tourny, "stroking characteristics in freestyle swimming and relationship with anthropometric characteristics", Journal of Applied Biomechanics, vol. 12, no. 2, pp. 197 206, 1996.

[15] A. Gustafsson y P. Lindenfors, "Human size evolution : No evolutionary allometric relationship between male and female stature," Journal of Human Evolution, vol. 47, no. 4, pp. 253 - 266, 2004.

[16] S. Bojanini, "Diseño antropométrico de un puesto de trabajo", Revista Universidad EAFIT, vol. 62, pp. 119131, 1986.

[17] E. McCormick y M. sanders, Human factors in engineering and design, New York: McGraw Hill, 1987.

[18] I. Gentil, "Podología preventiva: síndrome de espalda dolorosa en el podólogo", Revista Internacional de Ciencias Podológicas, vol. 2, no. 2, pp. 39 - 47, 2008.

[19] M. M. Galán, “Análisis cinemático de sistema mecánico con control giroscópico para ascenso y descenso de escaleras en una silla de ruedas para pacientes hemipléjicos con lesiones torácicas en t4 y menores", tesis doctoral, Instituto Politécnico Nacional, Ciudad de Mexico, 2010.

[20] J. Estrada, J. Camacho y M. T. Restrepo, "Parámetros antropométricos de la población laboral colombiana, 1995", Revista de la Facultad Nacional de Salud pública, vol. 15, no. 2, pp. 112139, 1998.

[21] G. Wheatley, "Toma de decisiones", 2012. [En línea]. Available: http:// mroad.nsinfo.hu/ckfinder/userfiles/ files/MROAD_LO 3 ES.pdf. [Accedido: 30-sep-2016]

[22] Dewert, «http://www.dewert.de/,» 2015. [En línea]. Available: http:// www.dewert.de/productos/cat-logos/ actuadores-lineales/. [Accedido: 2-oct2016]

3] A. Bermejo, "Ayudas para la marcha en la parálisis cerebral infantil", Revista Internacional de Ciencias podológicas, vol. 6, no. 1, pp. 9 - 24, 2012.

[24] E. García Diez, "Fisioterapia de la espasticidad: técnicas y métodos", Fisioterapia, vol. 26, no. 1, pp. 25 - 35, 2004. 
[25] K. Quiñones, A. Robayo y A. García, "Diseño de un prototipo de bipedestador para pacientes pediátricos con espina bífida", Revista Facultad de Medicina, vol. 61, no. 4, pp. 423 - 429, 2013.

[26] P. Cares, "Silla de ruedas dual, posibilitando la sedestación y la bipedestación infantil", Universidad Católica de Temuco, Escuela de Diseño, Temuco, Chile, 2005.

[27] L. Gongora, C. Rosales, I. González y N. Pujals, "Articulación de la rodilla y su mecánica articular", Medisan, vol. 7, no. 2, pp. 100 - 109, 2003.

[28] M. Latarjet, A. Ruiz Liard y E. Pró, Anatomía Humana, Buenos Aires: Editorial Médica Panamericana, 2004.

[29] M. Prives y N. Bushkovich, Anatomía Humana, Moscú: Mir, 2009.

[30] M. Bodor, "Quadriceps protects the cruciate ligament", Journal of Orthopaedic Research, vol. 19, no. 4, pp. 629 - 633, 2001.

[31] M.A. Diéz y J. Couceiro, "Problemas mecánicos de la rodilla", Revista Española de Cirugía Ortopédica y Traumatología, vol. 42, no. 1, pp. 5361, 1998.

[32] Z. Matjačić, A. Olenšek y T. Bajd, "Biomechanical characterization and clinical implications of artificially induced toe-walking: Differences between pure soleus, pure gastrocnemius and combination of soleus and gastrocnemius contractures", Journal of Biomechanics, vol. 39, no. 2, pp. 255 - 266, 2006.

[33] E. Avallonae T. Baumeister, Manual del Ingeniero Mécanico, México: MacGraw Hill, 2010.

[34] Gerdau-Corsa, «http://www.acesta. com.mx/,» 2014. [En línea]. Available: http://www.acesta.com.mx/boletin/5/ Grados_Tipos_Aceros.pdf. [Accedido: 10-nov-2016]

[35] A. González y E. Martínez, "Silla para asistencia en la higiene de personas con discapacidad", Trabajo final de grado, Universidad Politécnica de Cataluña, Cataluña, 2014.

[36] M. Romero, P. Museros, M.D Martínez y A. Poy, Resistencia de Materiales, Castelló: Universitat Jaume, 2003.

[37] R. Budynas y K. Nisbett, Shigley's Mechanical Engineering Design, U.S.A: McGraw Hill, 2012.

[38] J. Bernal, Vigas, Buenos Aires: Nobuko, 2005 . 